\title{
Reconstitution of a new Cysteine biosynthetic pathway in Mycobacterium tuberculosis
}

Kristin E. Burns, Sabine Baumgart, Pieter C. Dorrestein, Huili Zhai, Fred W.

McLafferty, Tadhg P. Begley*

Department of Chemistry and Chemical Biology, Cornell University, Ithaca, NY 14853

Supporting Information

\section{Experimental}

General Information: All reagents were purchased from Sigma-Aldrich.

Cloning, Expression and Purification of CysO, CysM: M. tuberculosis Rv1335 and Rv1336 were cloned into pET16b vectors, overexpressed in E. coli in LB at reduced temperature, and purified by Ni-Nta chromatography. The proteins were desalted and concentrated prior to use.

Cloning, Expression and Purification of CysO-thiocarboxylate: The CysO-intein construct was cloned according to manufacturer's instructions with a pTYB1 vector. It was overexpressed in E. coli at reduced temperature and purified using Chitin resin. Following elution with $\left(\mathrm{NH}_{4}\right) \mathrm{S}$ for 40 hours at $4^{\circ} \mathrm{C}$, the protein was desalted and concentrated.

Cloning, Expression and Purification of Mec ${ }^{ \pm}:$The plasmid pET-MAL-HT (6 histidine residues at the carboxy terminus of maltose binding protein) was used to clone Rv1334. It was overexpressed in E. coli at reduced temperature and purified by Ni-Nta chromatography. The protein was desalted and concentrated prior to use.

Cloning, Expression and Purification of $\mathrm{His}_{10}$-CysO-cys: A cysteine residue was added to the carboxy terminus of CysO by adding TGC bases to the primer before the stop codon. The construct was expressed and purified similarly to CysO.

CysO-cysteine formation by ESI-MS: $13 \mathrm{mM}$ O-acetylserine, $300 \mu \mathrm{g}$ CysOthiocarboxylate, and $6.7 \mathrm{mM}$ DTT were incubated with $480 \mu \mathrm{g}$ CysM in $50 \mathrm{mM}$ Tris-HCl (pH 8.0). After incubation at $37^{\circ} \mathrm{C}$ for certain time points, the samples were frozen at $80^{\circ} \mathrm{C}$. The samples were analyzed by ESI-FTMS.

Cleavage of $\mathrm{His}_{10}$-CysO-cys by $\mathrm{Mec}^{+}$: $255 \mu \mathrm{g}$ of $\mathrm{His}_{10}$-CysO-cysteine, $10 \mathrm{mM}$ DTT, $10 \mu \mathrm{M} \mathrm{ZnCl}_{2}$, and $45 \mu \mathrm{g} \mathrm{Mec}^{+}$were incubated in $50 \mathrm{mM}$ Tris, $\mathrm{pH} 8$ for 1 hour, then frozen. Samples were analyzed by ESI-FTMS. 
Ninhydrin assay for cysteine: Briefly, $6 \mathrm{mM}$ O-acetylserine, $10 \mathrm{mM}$ DTT, $250 \mu \mathrm{g}$ CysOthiocarboxylate, $100 \mu \mathrm{g}$ CysM, $0.1 \mathrm{mM} \mathrm{Zn}^{2+}$, and $140 \mu \mathrm{g} \mathrm{Mec}^{+}$were incubated in $100 \mathrm{mM}$ Phosphate buffer, $\mathrm{pH}$ 8.0. Samples were withdrawn at appropriate times, ninhydrin was added, boiled for 10 minutes, cooled, and ethanol added. Absorbance at 560nm was measured.

Copurification of CysO and MoeZ: CysO and MoeZ were expressed in E. coli at reduced temperature. The cell pellets were combined in lysis buffer and sonicated. Purification proceeded according to Ni-Nta protocol. The sample was desalted and concentrated prior to analysis by ESI-FTMS. 
